\title{
Toward Automated Optimization of Reconstruction of Atom Probe Data
}

\author{
D.J. Larson, B.P. Geiser, T.J. Prosa and T.F. Kelly
}

Cameca Instruments Inc., 5500 Nobel Drive, Madison, WI 53711

Atom probe tomography (APT) [1] is uniquely capable of measuring compositional features at the atomic scale. For the case of thin-film analyses [2], it is generally true that the elements or phases in the films of interest have different evaporation fields, and this results in curvature variations on the specimen endform [3-5]. As current reconstruction methods [6-8] do not account for these curvature variations, it is desirable to have a method to optimize the reconstruction parameters based on the nature of the data itself. In this paper we present simulated field evaporation data [9] as 'truth' and minimize positioning errors in the reconstruction automatically as a first step in developing a process to use on real data. The simulated volume contains an interface oriented with its normal parallel to the specimen axis. The advantage of using simulated data is that we have knowledge of the preevaporated $(\mathrm{x}, \mathrm{y}, \mathrm{z})$ positions of each atom and may compare these original positions with reconstructed positions while varying the reconstruction parameters. The main input parameters to the reconstruction are the standard initial radius $\left(\mathrm{R}_{0}\right)$, shank semi-angle $(\alpha)$ and image compression factor (ICF) (also equal to the inverse of the angular magnification factor) [4-8]. By demonstrating this automated method on simulated data, we learn how to proceed with real data and we can test the effectiveness against a known result.

For the simulated evaporation, the initial shape of the specimen was $R_{0}=20 \mathrm{~nm}$ and $\alpha=5^{\circ}$ with a facecentered cubic lattice parameter of $0.4 \mathrm{~nm}$ and a density of 60 atoms $/ \mathrm{nm}^{3}$ (equivalent to aluminum). Using these values (and $\mathrm{ICF}=1.4$ ) as an initial guess for the global reconstruction parameters produces the atom map shown in Fig. 1a. The corresponding normalized density metric (KNN metric [10] with $\mathrm{K}=20$ ) and planarity are shown in Figs. 1b and 1c. Both of these metrics may be used independently to optimize the reconstruction, as discussed below.

Note that the dashed lines in Fig. 1c correspond to the thickness of the atom map shown in Fig. 1a.

The data shown in Fig. 2 are the result of optimization of the global reconstruction parameters (results $\mathrm{R}_{0}=27 \mathrm{~nm}, \alpha=6.5^{\circ}, \mathrm{ICF}=1.25$ ) with respect to minimization of the root mean square of the deviation of the reconstructed $(\mathrm{x}, \mathrm{y}, \mathrm{z})$ compared to the original $(\mathrm{x}, \mathrm{y}, \mathrm{z})$. Further optimization of ICF functions [5], allowing variation along the detector radial hit position using the same optimization approach, produces the data shown in Fig. 3. Note the near elimination of the low density region $(<100>$ pole $)$ in the center of the image shown in Fig. 2a (arrowed in the distribution Fig. 2b).

In real data however, we cannot use the known $(\mathrm{x}, \mathrm{y}, \mathrm{z})$ values as we do not have them, but we can optimize the reconstruction parameters by minimization of the interface planarity shown in Fig. 1c. The result is shown in Fig. 4 where we have not used any a priori knowledge of the atomic positions prior to evaporation, but only assumed ICF functions as previously determined empirically [5] and confirmed in the current work. The global parameters were determined to be $\mathrm{R}_{0}=23.4 \mathrm{~nm}, \alpha=5.2^{\circ}$, and $\mathrm{ICF}=1.4$. The results shown in Fig. 4 are similar in quality to the results shown in Fig. 3 which used knowledge of the known $(\mathrm{x}, \mathrm{y}, \mathrm{z})$ positions to determine the reconstruction parameters.

[1] M.K. Miller et al., Atom Probe Field Ion Microscopy, Oxford University Press, Oxford, 1996.

[2] D.J. Larson et al., MRS Bulletin 34 (2009) 732.

[3] F. Vurpillot et al., Microsc. Microanal. 10 (2004) 384.

[4] E.A. Marquis et al., J.Microscopy (2011) doi: 10.1111/j.1365-2818.2010.03421.x

[5] D.J. Larson et al., J.Microscopy (2011) doi: 10.1111/j.1365-2818.2010.03474.x 
[6] P. Bas et al., Appl. Surf. Sci. 87/88 (1995) 298.

[7] B. P. Geiser et al., Microsc. Microanal. 15(S) (2009) 292CD.

[8] B. Gault et al., Ultramicroscopy (2011) doi: 10.1016/j.ultramic.2010.11.016.

[9] B. P. Geiser et al., Microsc. Microanal. 15(S) (2009) 302CD.

[10] L. T. Stephenson et al., Microsc. Microanal. 13 (2007) 448.
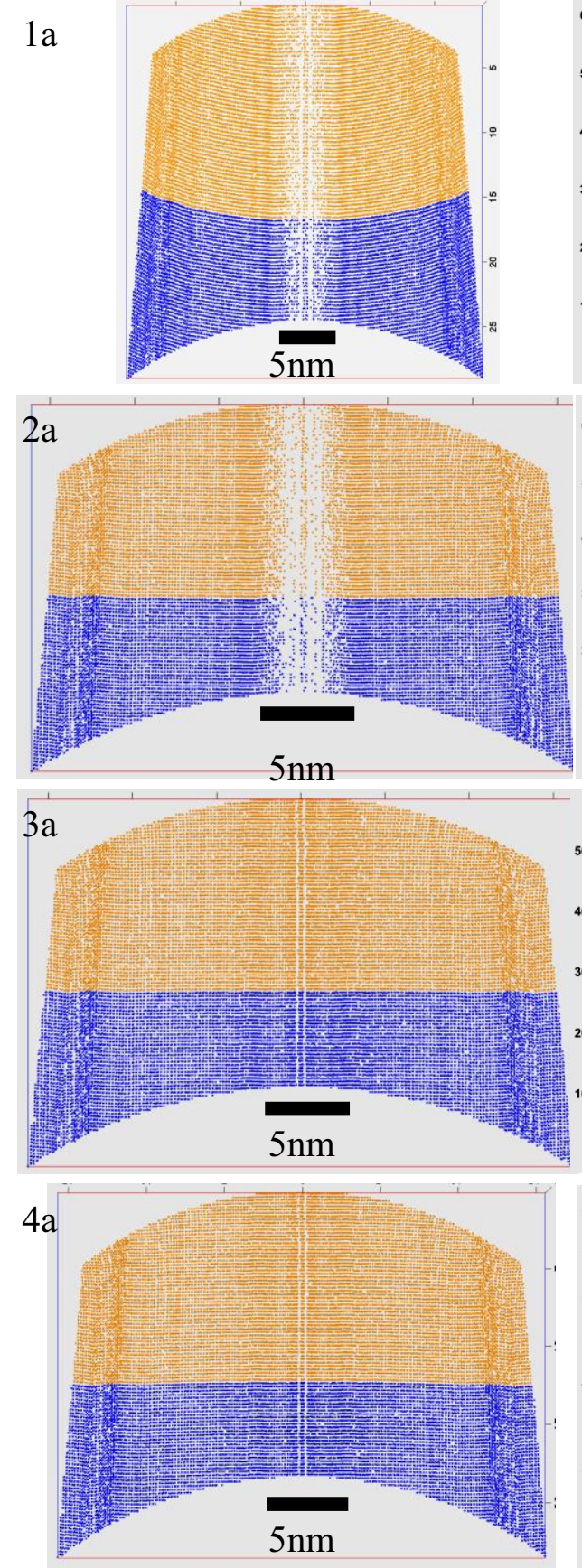
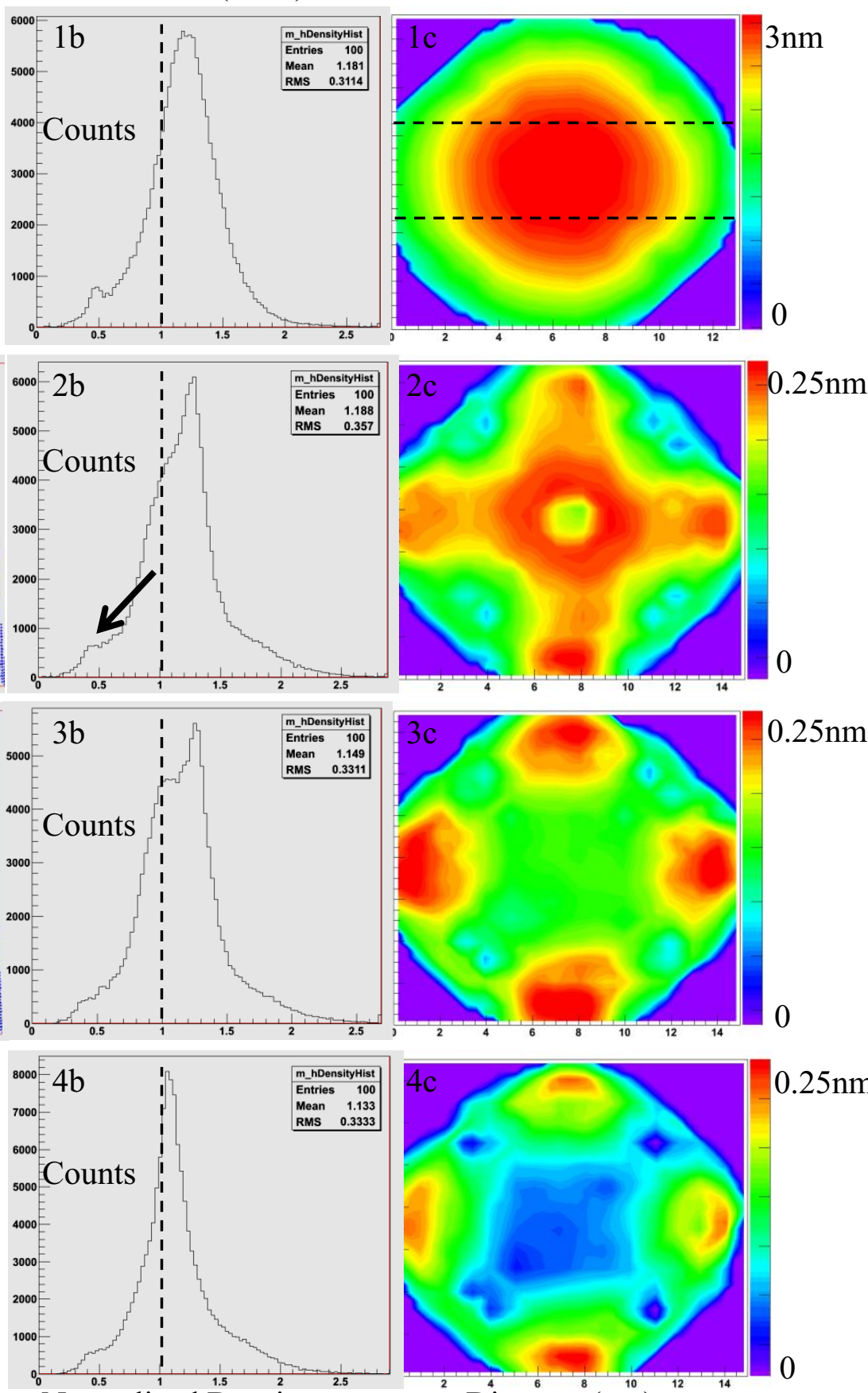

Normalized Density

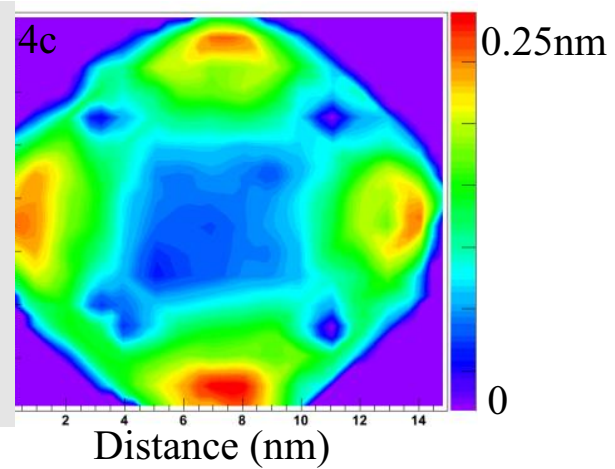

Fig. 1. (a) atom map, (b) density metric and (c) planarity for $\mathrm{R}_{0}=20 \mathrm{~nm}, \alpha=5^{\circ}$, and $\mathrm{ICF}=1.4$.

Fig. 2. (a) atom map, (b) density metric and (c) planarity $\mathrm{R}_{\mathrm{o}}=27 \mathrm{~nm}, \alpha=6.5^{\circ}$, and $\mathrm{ICF}=1.25$.

Fig. 3. Same as Fig. 2 using optimized ICF functions (seven points in detector radius space).

Fig. 4. (a) atom map, (b) density metric and (c) planarity for planarity-optimized global parameters using ICF functions from Fig. $3 \mathrm{a}$. 